\title{
Enhanced chiral sensing with dielectric nano-
}

\section{resonators}

Jose García-Guirado ${ }^{1}$, Mikael Svedendahl ${ }^{2}$, Joaquim Puigdollers ${ }^{3}$, and Romain Quidant ${ }^{1,4}$

1 - ICFO-Institut de Ciències Fotòniques, The Barcelona Institute of Science and Technology, 08860 Castelldefels (Barcelona), Spain.

2 - KTH Royal Institute of Technology, Roslagstullsbacken 21, 10691 Stockholm, Sweden.

3 - Universitat Politècnica de Catalunya (UPC), Departament d’Ingeniería Electrónica, 08034

Barcelona, Spain.

4 - ICREA-Institució Catalana de Recerca I Estudis Avançats, 08010 Barcelona, Spain.

\section{Supporting Information}

\section{CONTENTS}

This supporting information covers the following sections:

1. Numerical calculations of chiral media

2. Comparison with analytical theory

3. Additional FEM simulations

4. Fabrication process

5. Optical measurements

6. Reproducibility studies 


\section{Numerical calculations of chiral media}

Transmission spectra and near fields of arrays of Si cylinders were conducted using the COMSOL FEM software. The quartz substrate was modelled as a dielectric with refractive index 1.5 , the molecular layer had the refractive index 1.6 $+0.001 \mathrm{i}$, and the Si refractive index was taken from ellipsometric measurements of the layers used in the measurements.

The implementation of chiral media was conducted following previous works, where the equations in COMSOL were modified to reflect

$$
\begin{gathered}
\bar{D}=\varepsilon_{0} \varepsilon_{r} \bar{E}-\frac{i \kappa \bar{H}}{c} \\
\bar{H}=\frac{\bar{B}}{\mu}+\frac{i \kappa \bar{E}}{c},
\end{gathered}
$$

where $\bar{D}$ is the electric displacement field, $\bar{E}$ is the electric field, $\varepsilon_{0}$ and $\varepsilon_{r}$ are the vacuum and relative permittivities, $\kappa$ is the chiral parameter, $\mathrm{c}$ is the speed of light in vacuum, $\mu$ the permeability $\bar{H}$ the magnetic field and $\bar{B}$ the magnetic flux density. $\kappa$ is a function of wavelength and chiral molecules generally have a resonance response in the UV spectral range. $\kappa$ is, however, weak in the visible and near-infrared spectral range and varies slowly with the wavelength. Therefore, we modelled the coating with a constant $\kappa$ over the simulated wavelengths, typically with values $\kappa= \pm(1+0.01 i) \cdot 10^{-4}$, unless otherwise stated.

\section{Comparison with analytical theory}

The numerical results were verified by a comparison to analytical results. The transmission through a chiral slab can be calculated as: ${ }^{1,2}$ 


$$
\begin{aligned}
& T^{++}=\frac{4 n_{l} / n_{a} e^{-i d k_{0}\left(n_{l}-\kappa\right)}}{\left(n_{l} / n_{a}+1\right)^{2}-\left(n_{l} / n_{a}-1\right)^{2} e^{-2 i n_{l} k_{0} d}} \\
& T^{--}=\frac{4 n_{l} / n_{a} e^{-i d k_{0}\left(n_{l}+\kappa\right)}}{\left(n_{l} / n_{a}+1\right)^{2}-\left(n_{l} / n_{a}-1\right)^{2} e^{-2 i n_{l} k_{0} d}}
\end{aligned}
$$

, where $T^{++}$and $T^{--}$denotes transmission of $\sigma^{+}$and $\sigma^{-}$light respectively. Furthermore, the $n_{l}$ is the refractive index of the chiral layer, $n_{a}$ is the surrounding refractive index, $d$ is the chiral slab thickness, $k_{0}$ the wave number of light in vacuum, and $\kappa$ is the chiral parameter. This yields the following expression for the CD:

$$
C D=-\operatorname{atan}\left(\tanh \left(k_{0} d \operatorname{Im}[\kappa]\right)\right)
$$

Figure S1 show a comparison between numerical results using COMSOL simulations and the expressions above. Overall, we found an excellent agreement for all tests made. Figure S4a show the implementation of the real part of $\kappa$, and how it influences the phase of transmitted light. The real part does not affect the transmittance of chiral layers, which instead is governed by the imaginary part. Figure S1b-c show how the transmittance of a chiral slab is affected by the thickness of the slab.
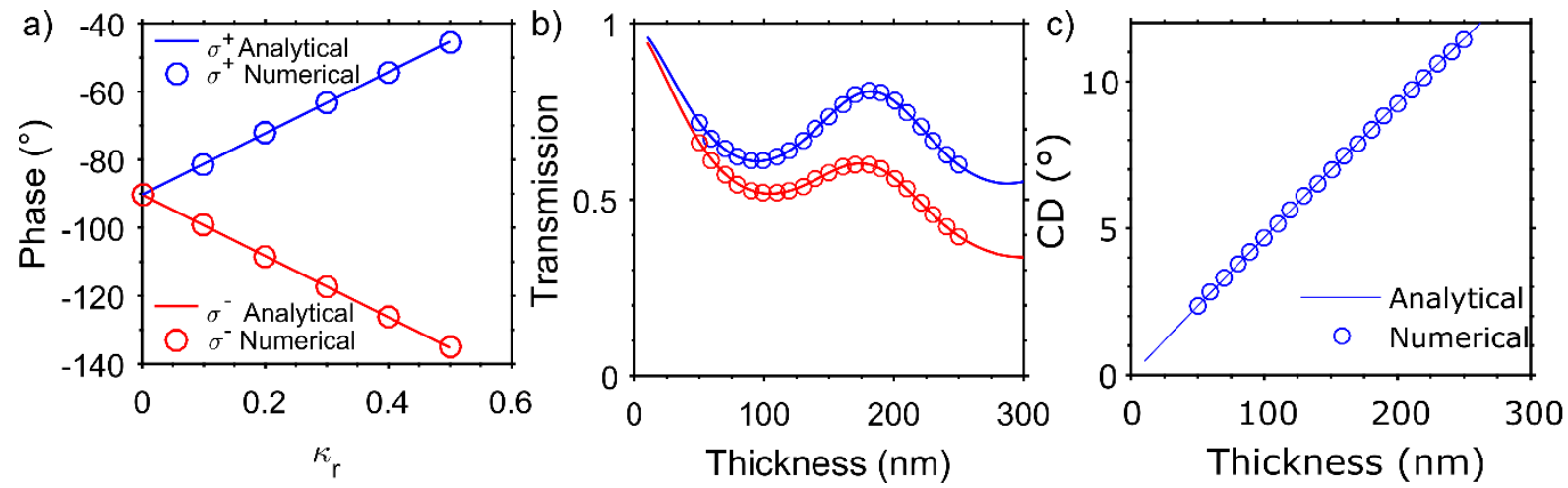

Figure S 1. Comparison of the numerical simulations and analytical solutions. A) The phase of circularly polarized light transmitted through a chiral slab of thickness $d=200 \mathrm{~nm}, \operatorname{Im}[\kappa]=0.05$, 
and refractive index $n_{l}=2+0.01 i$ as function of $\operatorname{Re}[\kappa]$. B) The transmission and c) the $C D$ of a chiral slab with $n_{l}=2+0.01 i$ and $\kappa=0.1-0.05 i$ as function of slab thickness, $d$. Calculations are made for a) $800 \mathrm{~nm}$ light and b-c) $765 \mathrm{~nm}$ light.

\section{Additional FEM simulations}

Figure S2-4 shows the electrical field enhancement, the magnetic field enhancement and the optical chirality enhancement for Si nano-cylinders of diameter $140 \mathrm{~nm}, 160 \mathrm{~nm}, 180 \mathrm{~nm}, 200 \mathrm{~nm}$, $240 \mathrm{~nm}$, and $280 \mathrm{~nm}$ at mid-height of the nano-cylinder. Figure S2-3 shows that the electrical and magnetic fields are equally enhanced regardless of the illumination handedness. This is important, as any chiral (or achiral) molecule will thus be interacting with the same field strength. Figure S4, on the other hand, show that the illumination handedness determines the optical chirality near the Si nano-structures. The magnitude of the optical chirality is nonetheless maintained and the difference in the optical chirality depending on illumination handedness is significant and uniform near the Si nano-structure.

a)

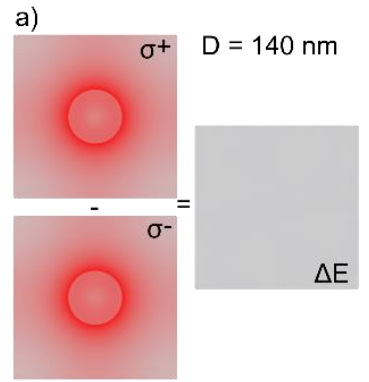

d)

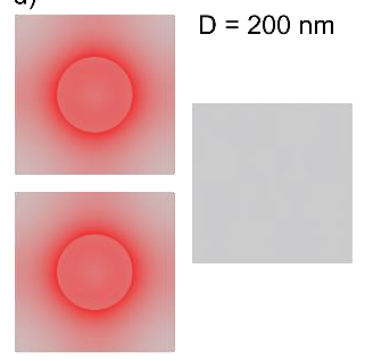

b)

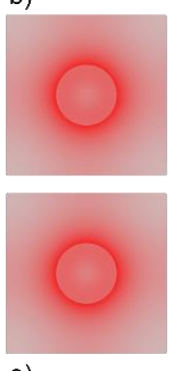

e)

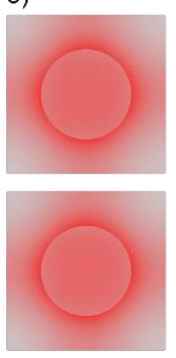

c)

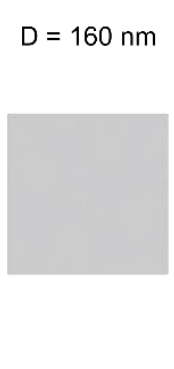

$D=240 \mathrm{~nm}$

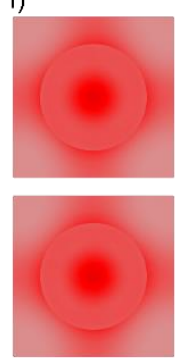

f)

$\mathrm{D}=180 \mathrm{~nm}$
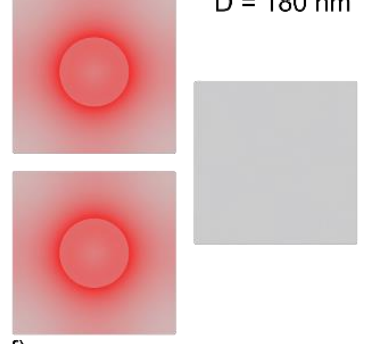

6

4

2

0

$\mathrm{D}=280 \mathrm{~nm}$

$-2$

$-4$ 
Figure S 2. Electric field enhancement and difference under $\sigma+$ and $\sigma$-illumination for a Si nanocylinder array with period $420 \mathrm{~nm}$, height $130 \mathrm{~nm}$, diameter according to the illustration and light illuminated at a) $745 \mathrm{~nm}$, b) $785 \mathrm{~nm}$, c) $820 \mathrm{~nm}$, d) $845 \mathrm{~nm}$, e) $890 \mathrm{~nm}$ and f) $890 \mathrm{~nm}$.

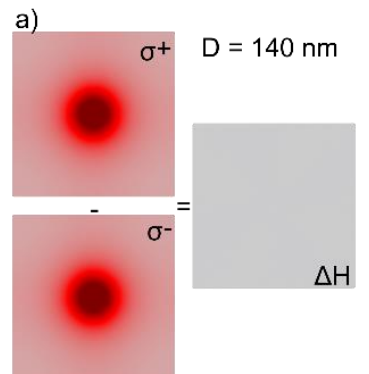

d)

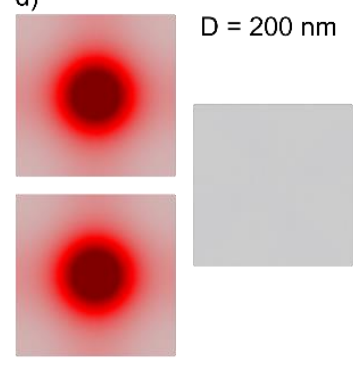

b)

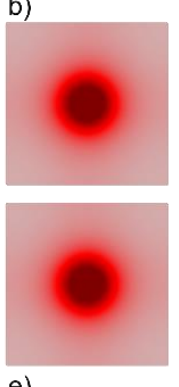

e)

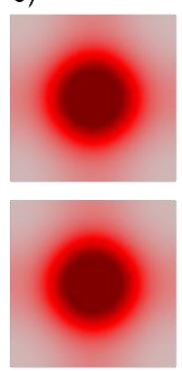

c)
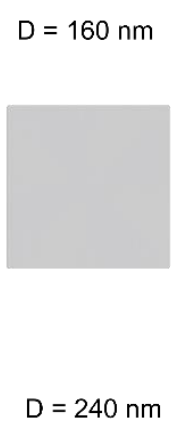

$D=240 \mathrm{~nm}$
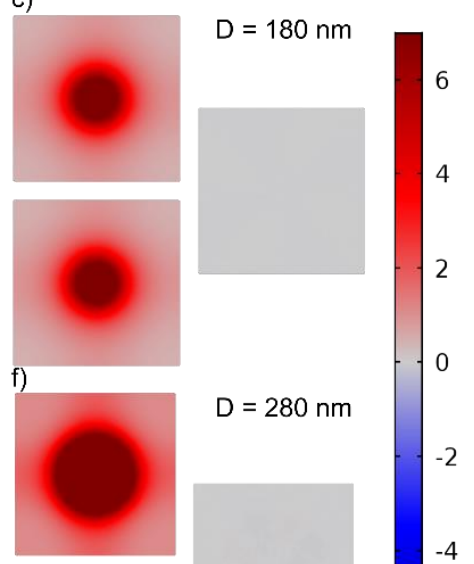

$D=280 \mathrm{~nm}$

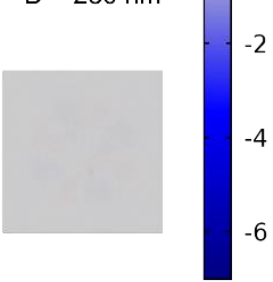

Figure S 3. Magnetic field enhancement and difference under $\sigma+$ and $\sigma$-illumination for a Si nanocylinder array with period $420 \mathrm{~nm}$, height $130 \mathrm{~nm}$, diameter according to the illustration and light illuminated at a) $745 \mathrm{~nm}$, b) $785 \mathrm{~nm}$, c) $820 \mathrm{~nm}$, d) $845 \mathrm{~nm}$, e) $890 \mathrm{~nm}$ and f) $890 \mathrm{~nm}$. 
a)

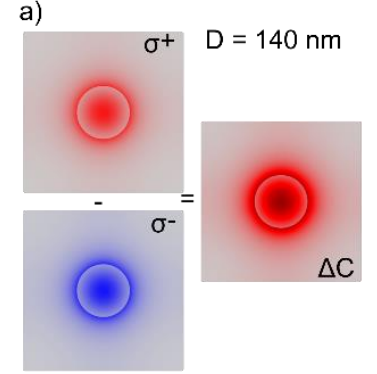

d)

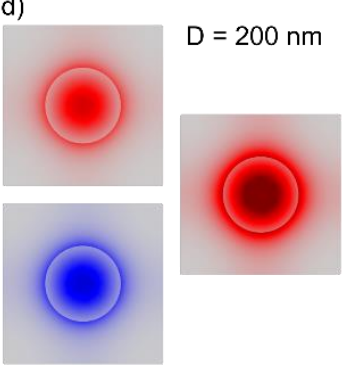

b)

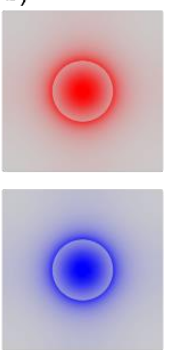

e)

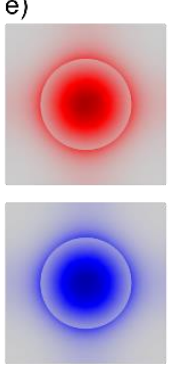

c)

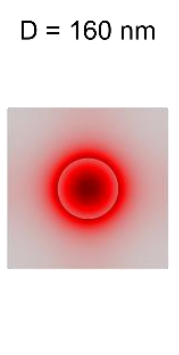

$D=240 \mathrm{~nm}$

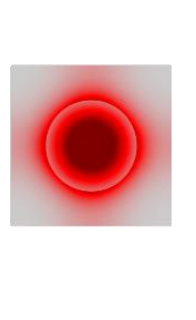

f)
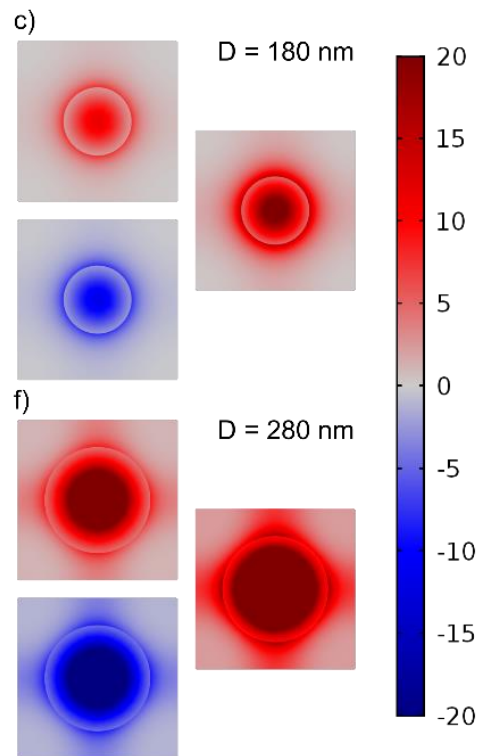

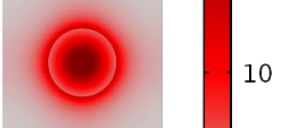

$D=280 \mathrm{~nm}$

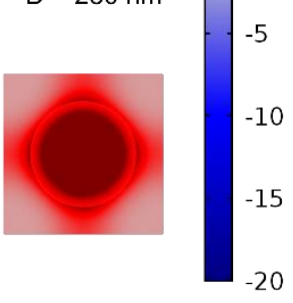

Figure S 4. Optical chirality enhancement and difference under $\sigma+$ and $\sigma$-illumination for a Si nano-cylinder array with period $420 \mathrm{~nm}$, height $130 \mathrm{~nm}$, diameter according to the illustration and light illuminated at a) $745 \mathrm{~nm}$, b) $785 \mathrm{~nm}$, c) $820 \mathrm{~nm}$, d) $845 \mathrm{~nm}$, e) 890 and f) $890 \mathrm{~nm}$.

Figure 5 show a comparison of the induced CD from a layer with real and imaginary $\kappa$, respectively. The bisignate lineshapes of the $\mathrm{CD}$ spectra induced by molecular layers containing real $\kappa$, is an indication that this part is dominant in the interaction with the nano-cylinders in this spectral range. 

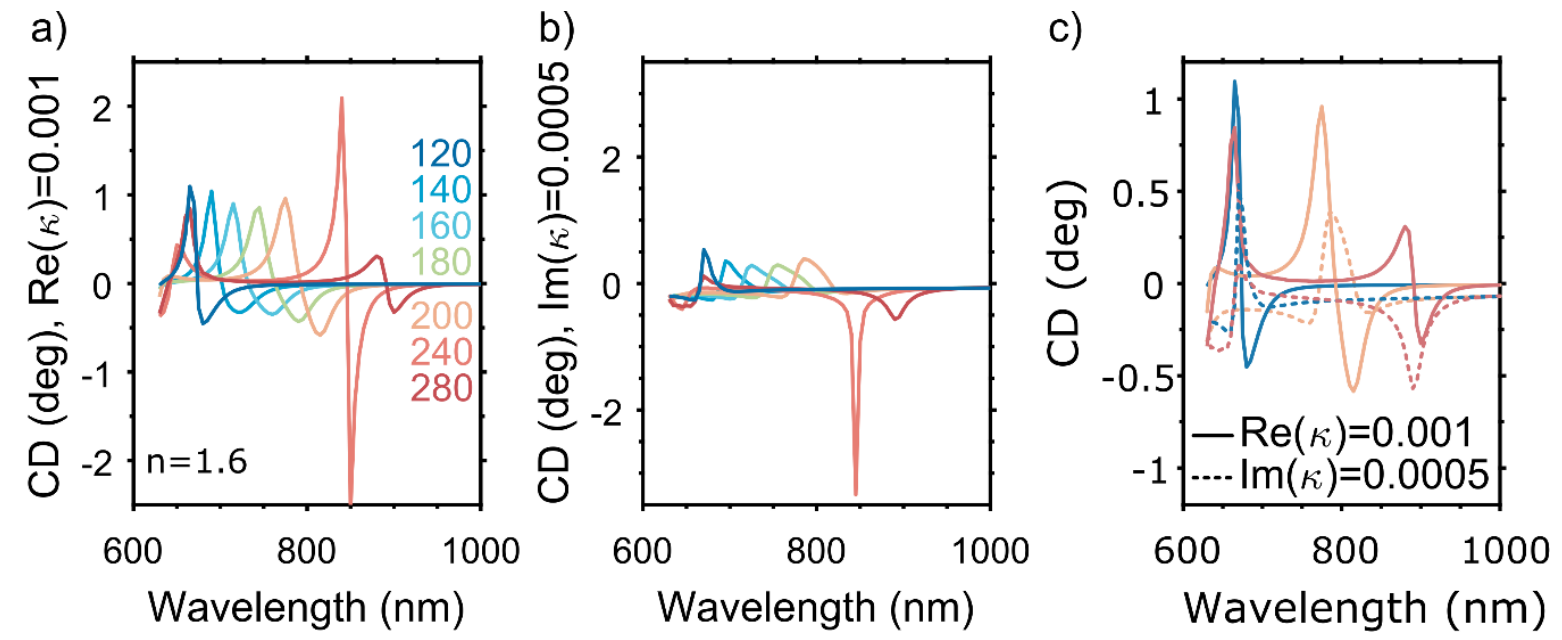

Figure S 5. The induced CD from a molecular layer, refractive index $n=1.6$, in Si nano-cylinders of diameters from 120-280 $\mathrm{nm}$ by a) a real-valued and b) imaginary chiral parameter, $\kappa . c$ ) a direct comparison of the lineshapes for diameters 120,200 and $280 \mathrm{~nm}$.

The loss inside the nano-structures is not chiral without the addition of a chiral layer. However, slight variations of the electric field, likely induced by array interactions, yields locally enhanced dissipation for either $\sigma^{+}$or $\sigma^{-}$polarization, which cancels when integrated throughout the nanoparticle volume (Figure S6a,d). When a chiral layer interacts with the nano-structure, additional chiral dissipation is found (Figure S6b-c,e-f). 


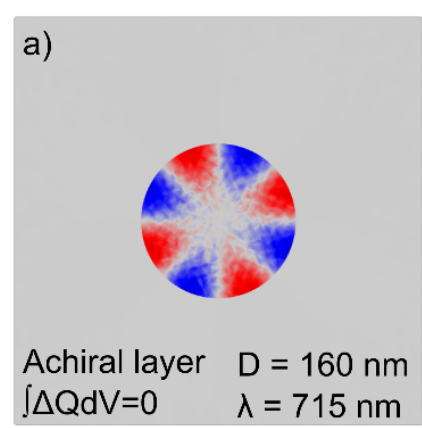

d)

$\triangle \mathrm{QdV}=0 \quad \lambda=715 \mathrm{~nm}$

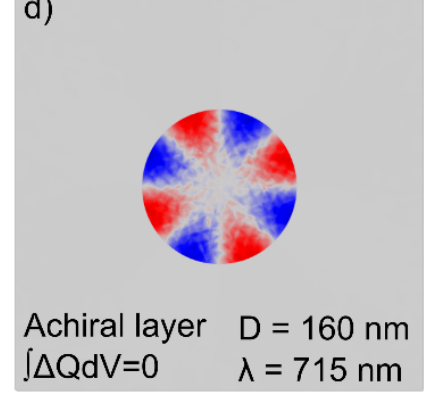

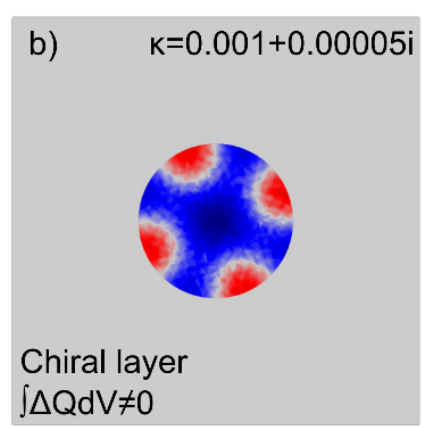

e) $\quad k=-0.001-0.00005 \mathrm{i}$

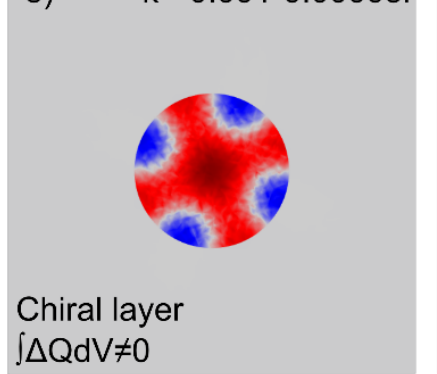

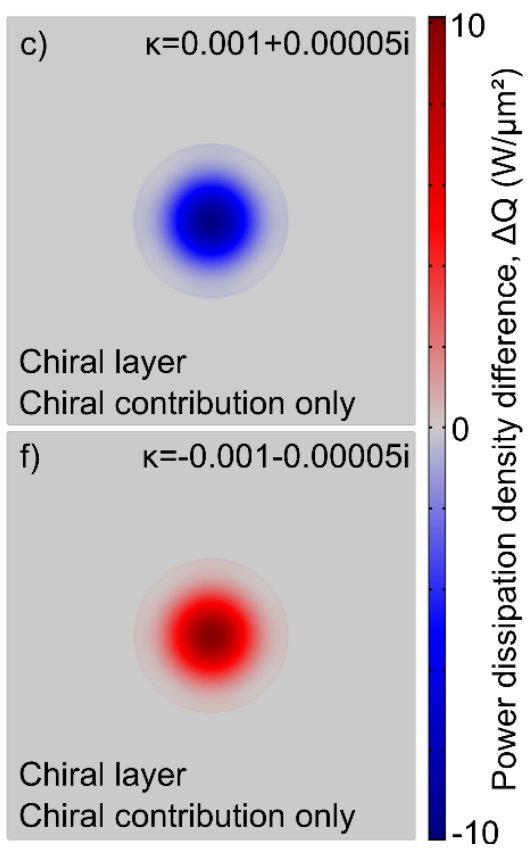

Figure $S$ 6. The power dissipation density difference for $D=160 \mathrm{~nm} h=130 \mathrm{~nm}$ cylinder surrounded by a,d) an achiral layer and b,e) a chiral layer. c,f) Same as (b,e), but the achiral signatures in $(a, d)$ are removed to visualize the chiral contribution to the loss. The chiral parameter $\kappa$ is positive in $(b-c)$ and negative in (e-f).

\section{Fabrication process}

The sensor consisted in an array of cylinders. The cylinders can be described by their diameter D and height $\mathrm{h}$ while the array is described by its period $\mathrm{P}_{\mathrm{x}}$ and $\mathrm{P}_{\mathrm{y}}$, and its size $\mathrm{S}$. In this particular case we use $\mathrm{P}_{\mathrm{x}}=\mathrm{P}_{\mathrm{y}}=\mathrm{P}$ to get $\mathrm{C} 4$ symmetry to prevent linear dichroism contributions in our signals ${ }^{3}$. The sensors were designed to have electric and magnetic dipolar resonances in the VIS-NIR spectral range, thus the Period was chosen to $\mathrm{P}=420 \mathrm{~nm}$. The size of the arrays are $\mathrm{S}=120 \mu \mathrm{m}$ for convenience of our optical measurements. Figure S7a show a schematic drawing of the sensor. 
The sample fabrication consisted in a negative lithography process involving electron beam lithography (EBL) and reactive ion etching (RIE) for the sensor fabrication, and molecular thermal evaporation for the deposit of the molecules. Four sensor samples were fabricated in parallel in the same substrate to prevent discrepancies from sequential sample fabrication (Fig. S7b). The procedure is described in the figure S7c. Substrates of $130 \mathrm{~nm}$ amorphous silicon coated on fused silica (Siegert Wafer $\mathrm{GmbH}$ ) were used as base for the sensors. The substrates were spin-coated with 80nm of ARN7500.08 (Allresists $\mathrm{GmbH}$ ) then exposed using the Crestec CABL 9510C EBL tool. Subsequently, the amorphous silicon layer was etch using the Oxford Plasmalab System 100 RIE-ICP with $\mathrm{C}_{4} \mathrm{~F}_{8}$ and $\mathrm{SF}_{6}$ gasses. Then residual resist was cleaned during 5 min. in $\mathrm{O} 2$ plasma (PVA TePla 300). Next, the sample was spin-coated with AZ520D protective coating (Microchemicals $\mathrm{GmbH}$ ) and diced (DISCO DAD321) in four individual samples. Afterwards, the samples were cleaned in acetone and IPA and dried with N2. Finally, the samples were clean in piranha solution $\left(4 \cdot \mathrm{H}_{2} \mathrm{SO}_{4}: 1 \cdot \mathrm{H}_{2} \mathrm{O}_{2}\right)$ for $5 \mathrm{~s}$ to remove any residue of resist. As a final step, each sample was exposed during $5 \mathrm{~min}$. to UV ozone and right after it was individually coated with 200 nm of L-, D- or racemic phenylalanine (Sigma Aldrich 78019, P1751 and 147966) using molecular thermal evaporation (MTE) with a homemade setup as described in our previous work ${ }^{4}$. In parallel to the sensors coating we also coated fused silica (FS) substrates.

The cylinders arrays were characterized using scanning electron microscopy (SEM) to retrieve their actual geometrical sizes and check their morphology. The cylinders were found to be slightly over etch, not perfectly circular from the top and to have a bit of lateral slope, thus the effective diameter at half of the height $\mathrm{h}$ was chosen for calculations and plot representations of data. These deviations from a perfect shape may introduce spectral artifacts that have to be considered during the signal processing. The figure S7d shows a picture of arrays. The molecular coatings were also 
characterized with SEM to retrieve their thickness and check the sensor coverage. The figure S7e shows a section picture of the coated sensors.

a)
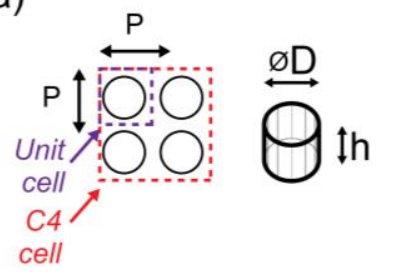

d)

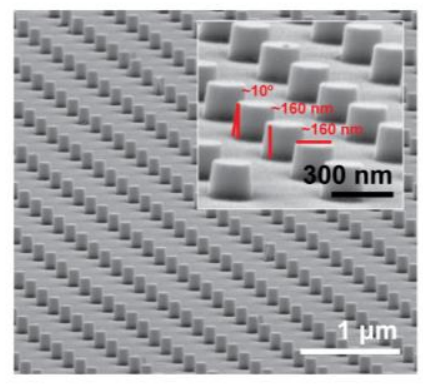

b)

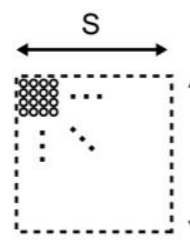

e)

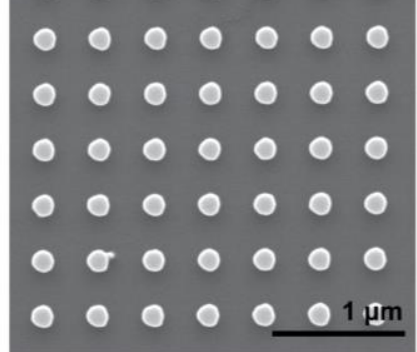

c)

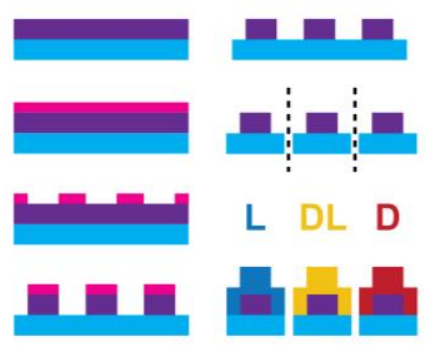

f)

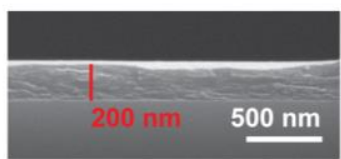

g)

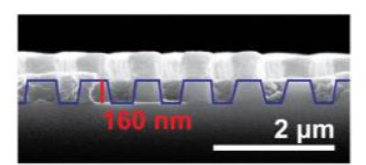

Figure S 7. a) layout of the cylinders and the sensor array, and b) layout of the four-replica sample. c) Fabrication and molecular coating process schematic. d,e) SEM images of the cylinders array sensor. f) SEM image of the section of the coatings and g) the coated sensors.

The molecular coatings were done using molecular thermal evaporation (MTE) as described in our previous work ${ }^{4}$. MTE provide a highly directional, homogeneous and dense deposit of randomly oriented molecules.

\section{Optical measurements}

The molecular coatings on FS were characterized in the ultraviolet UV range, were the molecules have their electronic resonances. The measurements were done using Chirascan Plus tool form 
Appliedphotophysics. The figure S8a shows the absorbance and CD spectra of the threephenylalanine coatings. The values of CD curves reveal the complementary and null contribution to $\mathrm{CD}$ of the enantiomers and the racemic mixture (figure $\mathrm{S} 8 \mathrm{~b}$ ). In order to guess the values of the $\mathrm{CD}$ of the coating at the visible (VIS) and near infrared (NIR) range, we fitted an exponential decay function to the experimental CD values (figure S8c). The extrapolation of the fitting toward the VIS NIR give us the values 0.98 and -0.66 mdeg for L- and D- coatings respectively. Although the spectra are phenomenological consistent, they turn to be slightly differences to our previous work, .These differences reinforces the need perform all the checkouts in this type of experiments.
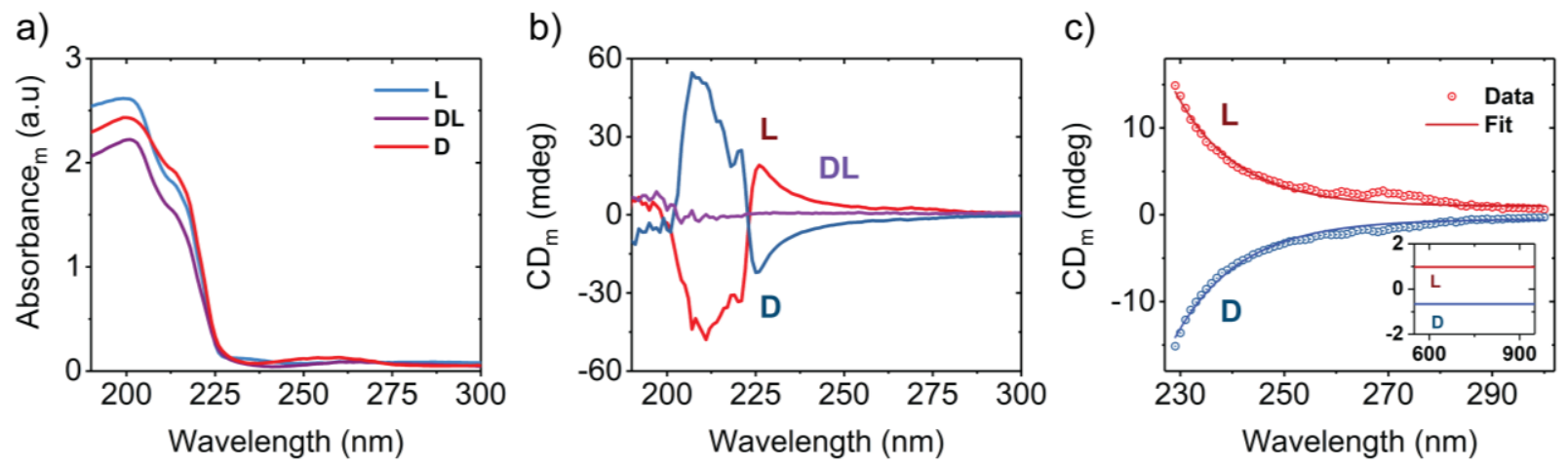

Figure S 8. UV spectral measurement of the phenylalanine coating on FS substrates. a) Absorbance, b) CD a c) fitting where the inset indicate the extrapolated values at the VIS-NIR range.

The measurements in the VIS-NIR spectral range were done using our homemade optical setup, which consist in a white light source and a broadband polarizing system to generate the excitation beam and a grating spectrometer to analyze the outgoing light from the sample ${ }^{4}$. Low NA objectives were used to focus and collect the light in order to minimize the distortion of polarization. The illuminated area and the collection area are centered and they are circular with 5 
$\mathrm{mm}$ and $60 \mathrm{um}$ of diameter respectively. The collection of light from the 120x120 um ${ }^{2}$ sensor arrays accounts for $\sim 20,000$ cylinder structures, more than enough to measure CD from nanostructure assemblies 5 . The four fabricated sample replicas allow us to measure the two enantiomers, the racemic mixture and keep one replica for uncoated measurements. Each replica may generally contain several sensor variations and/or geometries to account for ideal fabrication deviations (Fig 9a). The sensors of each replica were measured before the molecular coating to ensure spectral compatibility, i.e. the discrepancy between sensors. The figure S9b-c shows the extinction and CD spectra of the same uncoated sensor geometry for the four different replicas.

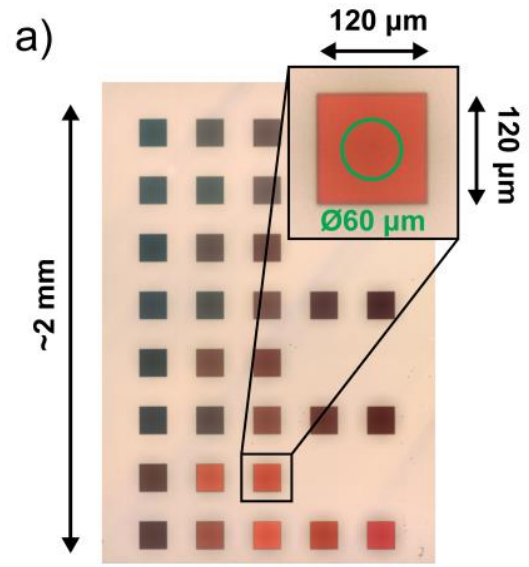

b)
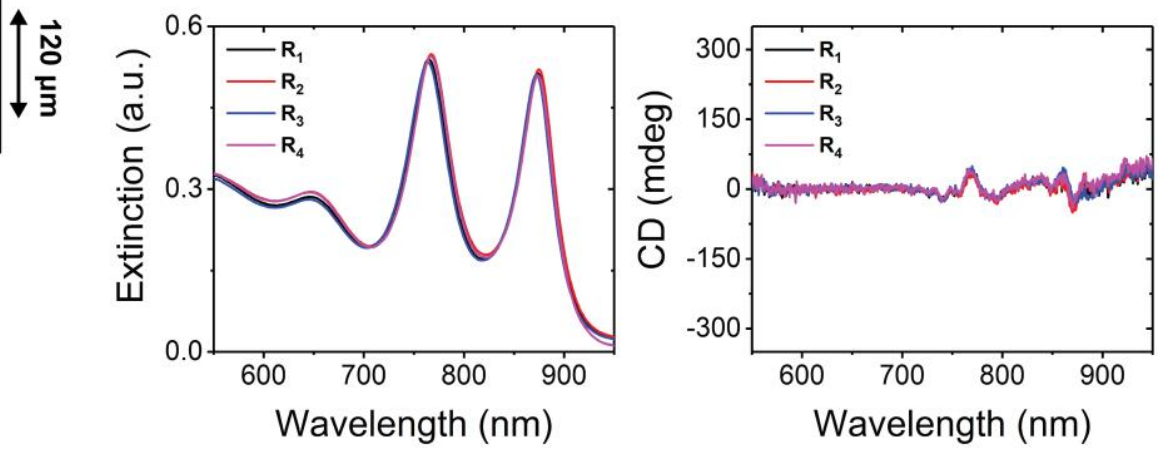

Figure S 9. a) Optical Image of full set of sensor variations included in one of the replicas. Inset: Single sensor array. The green circle depicts the size of the collection that limits the light that reaches de spectrometer. b) Example of extinction (left) and CD (right) spectra of the same uncoated sensor measured in all four sample replicas.

The sensor's spectra were acquired under left and right circularly polarized (LCP and RCP) light illumination, $\sigma^{-}$and $\sigma^{+}$respectively. Then, transmission signals $\mathrm{T}$, were used to calculate $\mathrm{CD}$ in mdeg as 


$$
C D=\tan ^{-1}\left(\frac{\sqrt{T_{\sigma^{-}}}-\sqrt{T_{\sigma^{+}}}}{\sqrt{T_{\sigma^{-}}}+\sqrt{T_{\sigma^{+}}}}\right) \cdot \frac{0.18}{\pi}
$$

where

$$
T_{i}=\frac{S_{i}}{R_{i}}, \quad i=\sigma_{-}, \sigma_{+}
$$

being $\mathrm{S}$ the transmitted light through the sensor and $\mathrm{R}$ the transmitted light through the bare substrate. Extinction signals are calculated as

$$
E_{i}=\log \left(\frac{1}{T_{i}}\right), \quad i=\sigma_{-}, \sigma_{+}
$$

For full transparency, we are going to describe our data processing protocols. In order to remove birefringence artifacts coming from fabrication defects and setup miss alignments, ninety degreerotated measurements were perform and averaged. As expected, the molecular coatings exhibited the complementary chiral signature in contrast to the zero CD of the uncoated sensors. However, the raw measurement rather than been perfect shows mainly the trend (Fig. S10a). At this step is where the racemic (DL-) coating plays and important role, since coatings equal concentration of the two enantiomers includes in its spectrum the possible bias of the real sensor toward any of the species, therefore after its subtraction to the enantiomers spectra a clear complementarity between L- and D- is revealed (Fig S10b). Nevertheless, there are still some factors associated to these measurements (relative sample tilt, rotation angles, etc.) that prevent the spectra to be ideal. For the shake of clarity, one can correct the clear base line deviation that distort the spectra shown in figure S10b to get signals that are more symmetrical in figure S10c. 
a)

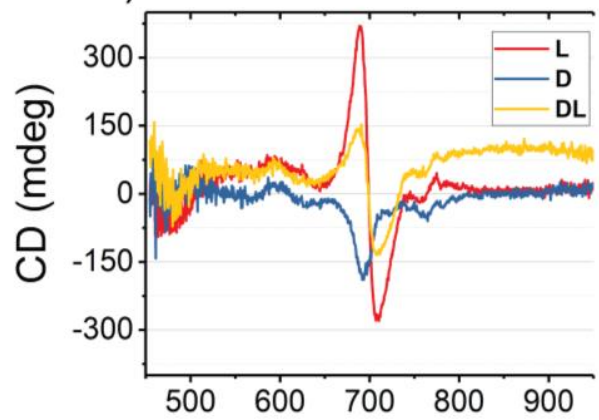

b)

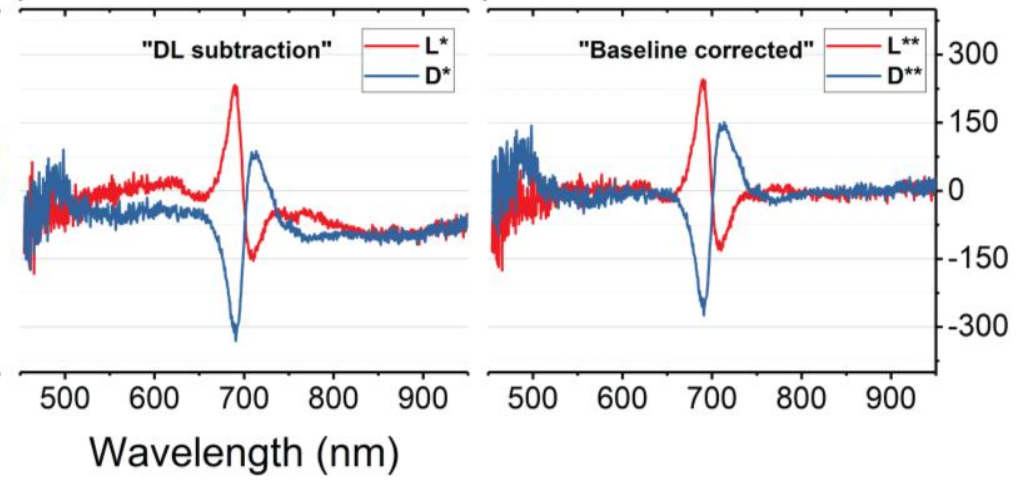

Figure S10: CD Signal processing example. a) Raw data coming from the tree coatings $L$ - and Denantiomers and DL-racemic mixture. b) After DL- spectrum subtraction to $L$ - and D-. c) After baseline deviation correction.

\section{Reproducibility studies}

In order to validate the measurements and face possible reproducibility issues, which have been topic of controversy in the fields for the last decade, we have repeated the same experiment up to 3 times from scratch. This essentially means that we have fabricated completely new batches of samples, made new molecular coatings, characterize and process the samples following exactly the same protocols. Our samples have always shown the same trends, although practice makes perfect. In figure S11a, we show the extinction and CD spectra for the same geometry from different batches. The small differences arise from subtle adjusts between batches during the fabrication process (fine dose adjustment, astigmatism corrections, etch rate, etc.). This result highlight the sensitivity to small sample changes of the CD signals.

Another important remark is about the boundaries of the sensing geometry. Our sensors showed similar CD enhancement for a variety of sensor geometries, i.e. cylinder diameters. However, this 
do not mean that a randomly chosen geometry would perform similar or give valid results. The figure S11b shows the spectra at the extremes of the studied range, where the symmetry of the CD signals were specially distorted. Out of the studied spectral range or for geometries slightly different, we found the CD signals extremely degraded. In these of plots, we highlight the change in the magnitude of the Bragg mode (BM), which was originally out our range of interest. It can be seen that the balance between the BM and the ED and MD changes when we sweep the cylinder diameter and it could be interfering as well with our measurements. With this, we want to point out subtle change in the sensor geometry (diameter, period, thickness, etc.) may induce unadvisable effects that could ruin the CD signals.
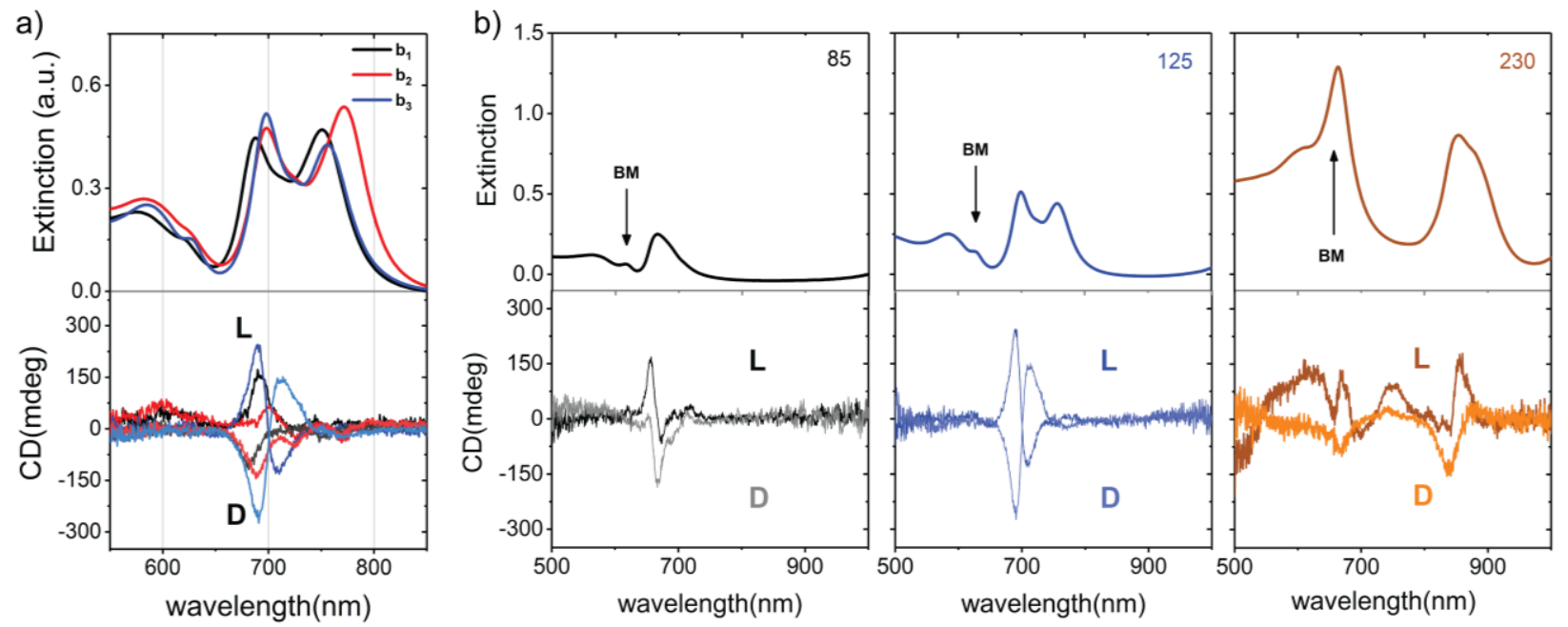

Figure S11: Experimental reproducibility. a) example of extinction (top) and CD (bottom) for a) the sample geometry form different fabrication batches $\left(b_{1}, b_{2}, b_{3}\right)$. b) Extinction (top) and $C D$ (bottom) for Silicon cylinder sensors of 85, 125 and $230 \mathrm{~nm}$ of diameter (left to right) coated with $200 \mathrm{~nm}$ of phenylalanine enantiomers (L- and D-). The Bragg mode (BM) peak is pointed with an arrow. 


\section{REFERENCES}

(1) Mohammadi, E.; Tsakmakidis, K. L.; Askarpour, A. N.; Dehkhoda, P.; Tavakoli, A.; Altug, H. Nanophotonic Platforms for Enhanced Chiral Sensing. ACS Photonics 2018, 5 (7), 26692675.

(2) Lindell, I. V; Sihvola, A.; Tretyakov, S. A.; Viitanen, A. J. Electromagnetic Waves in Chiral and Bi-Isotropic Media; Artech House Publishers: Boston, 1994.

(3) Hentschel, M.; Schäferling, M.; Weiss, T.; Liu, N.; Giessen, H. Three-Dimensional Chiral Plasmonic Oligomers. Nano Lett. 2012, 12 (5), 2542-2547.

(4) García-Guirado, J.; Svedendahl, M.; Puigdollers, J.; Quidant, R. Enantiomer-Selective Molecular Sensing Using Racemic Nanoplasmonic Arrays. Nano Lett. 2018, 18 (10), 6279_ 6285.

(5) Karst, J.; Strohfeldt, N.; Schäferling, M.; Giessen, H.; Hentschel, M. Single Plasmonic Oligomer Chiral Spectroscopy. Adv. Opt. Mater. 2018, 6 (14), 1-8. 